\title{
The Role of MR Arthrography in Evaluation of Femoroacetabular Impingement Syndrome
}

\author{
Mohamad Farouk Agag, Mahmoud Ibrahim Elshamy, Anas Abd Alaziz Ahmad*
}

Department of Radio-diagnosis, Faculty of Medicine - Al-Azhar University

*Correspondence author: Anas Abd Alaziz Ahmad; Mobile: (+20) 112926 2313; E-mail: anossof@gmail.com

\begin{abstract}
Background: Femoroacetabular impingement (FAI) is defined as an abnormal femoral acetabular contact that occurs within the normal range of motion caused by alterations of the acetabulum and/or the femoral head or neck. Objective: The purpose of this study was to compare the diagnostic performance of MR arthrography (MRA) with arthroscopic correlation for detecting labral tears and articular cartilage abnormalities. Materials and Methods: MRA was performed in 29 patients ( 16 males, 13 females with symptomatic FAI. The MRI images assessed for presence of labral tears, chondral lesions and osseous abnormalities. Arthroscopic correlation was obtained in all cases. Results: The sensitivity, specificity, PPV, and NPV of MRA for detecting labral tears were 87.5\%, 71.4\%, 91.3\% and 62.5\%, respectively. The sensitivity, specificity, PPV, and NPV of MRA for detecting chondral damage were 76.5\%, $91.7 \%$, 86.7\% and 78.6\% respectively. Conclusion: MRA appears to be an accurate imaging modality in the evaluation of labral tears. MRA is less accurate in the diagnosis of cartilage abnormalities in the hip.
\end{abstract}

Keywords: MR arthrography - Hip - FAI - Labral tears - Chondral lesions.

\section{INTRODUCTION}

The concept of impingement was reintroduced by

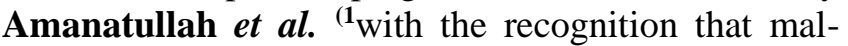
united femoral neck fractures that healed in retroversion could cause abnormal contact between the femoral neck and acetabular rim leading to accelerated posttraumatic osteoarthritis. Over the past 2 decades, 2.

Amanatullah et al. postulated that unrecognized developmental alterations and mal-orientations of the hip may be the underlying cause of primary or idiopathic hip osteoarthritis (2).

FAI is one of the causes of chronic hip pain, and it is an important factor in the development of osteoarthritis. Patients with femoroacetabular impingement are young ${ }^{(3)}$. Patients present with groin pain with hip rotation, in the sitting position, or during or after sports activities. Typically, they are aware of their limited hip mobility long before symptoms appear (4).FAI is divided into two types: cam and pincer, however, a mixed pattern is believed to often be present, with one of the two types predominating and it can be differentiated on the basis of a predominance of either a femoral or an acetabular abnormality ${ }^{(5)}$.

The role of imaging in FAI is to evaluate the hip for abnormalities associated with impingement. The main important imaging techniques for the assessment of FAI are conventional radiography, 3D computed tomography, and MR, especially MR arthrography ${ }^{(\boldsymbol{(})}$.

Conventional radiography: Is very useful for the identification of morphological bone alterations and to exclude arthritis, avascular necrosis or other joints problems. 3D computed tomography: 3D computed tomography represents an accurate tool to assess abnormalities of the femoral head-neck junction with moderate reliability. In addition, computed tomography with $3 \mathrm{D}$ surface rendering provides a virtual reality confirmation of the offset deformity for both the surgeon and patient, which is very difficult with 2D reconstruction. MRI: Nowadays is considered a good diagnostic tool in the preoperative assessment of the IBP joint, because of its accuracy in the evaluation of anatomic bone variants, and to assess the exact extent of the damage already present within the joint and of cartilaginous and labral injuries.

MR arthrography: Is considered the best imaging modality for routine evaluation of the internal hip pathology.

The most important advantages of this method include better visualization of the joint anatomy owing to easy differentiation of the joint surface and a higher soft tissue contrast obtained by intra-articular gadolinium dilution ${ }^{(7)}$. Arthroscopy: Is the current "gold standard" for diagnosing intra-articular hip joint pathological conditions and abnormalities ${ }^{(8)}$.

It is important to know that surgical treatment of FAI is only suitable in patients without advanced degenerative changes and without extensive articular cartilage damage, also it is important to identify the type of FAI because surgical treatment differs for each type. So, preoperative radiological assessment before such an invasive procedure is necessary for accurate diagnosis of acetabular labral tear and to rule out other abnormalities involving the hip joint ${ }^{(9)}$.

\section{AIM OF THE WORK}

The purpose of this study was to evaluate the diagnostic value of conventional MRI and MR arthrography in diagnosing patients with femoroacetabular impingement. We aimed to compare the MR arthrography results with hip arthroscopic findings (Gold Standard) to assess its diagnostic value in evaluating acetabular labral tears and articular cartilage defects.

\section{PATIENTS AND METHODS}

The study included 29 patients (16 male and 13 female), their ages ranged from 18 to 45 years with the median age of 31 years. Mean age was 30.9 years. 
ejhm.journals.ekb.eg

The study was performed in Al-Hussein Hospital, Faculty of Medicine, Al-Azhar University between October 2018 and June 2019.

The patients were referred to Radiology Department from the outpatient, inpatient and emergency units of the orthopedics department.

Ethical approval and written informed consent: An approval of the study was obtained from AlAzhar University Academic and Ethical Committee. Every patient signed an informed written consent for acceptance of the operation.

Methodology: All patients were subjected to the following:

- History taking.

- Clinical provisional diagnosis.

- Conventional MRI.

- MR arthrography.

- Surgical arthroscopy (done for all patients).

\section{MR Arthrography:}

- Direct MR arthrography using, fluoroscopic guided, CT guided, or ultrasound guided imaging.

1- Fluoroscopic guided:

- Using OMNI DIAGNOST MULTIPURPOSE X RAY SYSTEM PHILIPS which compromise a table with an over table, user interface, an automatic serial changer and a large image intensifier.

- Technique:

The area was prepared and draped in a sterile fashion. The subcutaneous tissue was anesthetized.

The needle tip 22 gauge was advanced in an anterior posterior until it reach femoral head neck junction.

Start injection of the contrast (the mixture used was composed of $1 \mathrm{mmol}$ gadopentetate dimeglumine, $5 \mathrm{ml}$ iodinated contrast, $3 \mathrm{ml}$ xylocaine and completed to 20 $\mathrm{ml}$ with sterile saline.)

The patient were scanned with MRI during maximally 30 min of injection.

2- CT guided:

- Using GE 4 DETECTORS MULTI-SLICE CT.

- Technique: Same technique as fluoroscopy.

3- Ultrasound guided:

- Using Ultrasound ALOKA PROSOUND ALPHA 7 or ALOKA PROSOUND ALPHA 6 (JAPAN) ultrasound device that equipped by $6-7 \mathrm{MHz}$ linear array transducer for musculoskeletal part probe.

- Technique: Same technique as fluoroscopy but iodinated contrast material is not used.

-

\section{Post intra-articular contrast injection MR sequences:}

- Coronal FAT-SAT post contrast

- Axial oblique T1 FATSAT post contrast

- Sagittal T1 FAT-SAT post contrast

\section{MR IMAGE INTERPRETATION}

Image interpretation using a standardized form in all cases.

\section{Conventional MRI:}

- For assessment of bone marrow edema and subchondral degeneration, detect osseous abnormality (head neck junction abnormality) and joint effusion.

\section{MR Arthrography:}

- Used for detection of type of impingement, labral tears and chondral lesions.

\section{Types of FAI:}

The type of femoroacetabular impingement was detected according to measures of alpha angle and acetabular depth.

Alpha angle measured in the axial oblique plane by:-

Placing a circle around the oblique axial circumference of the femoral head.

Placing a line passing through the narrowest portion of the femoral neck till the center of the femoral head.

Placing a second line that extends from the intersection of the first line and the center of the femoral head to the point where the osseous anterior femoral head intersect the circle.

Acetabular depth measured in the axial oblique plane by:-

1) Placing a line passing through the center of the femoral head.

2) A line connecting the anterior and posterior acetabular rim parallel to the first line.

The value is classified as "positive" if the center of the femoral head is lateral to the line that connects the anterior and the posterior acetabular rim and value is negative if the center of the femoral head is medial to the line which indicate deep acetabulum.

\section{Labrum}

The diagnostic criteria of labral tears was the high signal of contrast medium entered the inner of acetabular labrum or located between the acetabular labrum and acetabular edge or presence of an associated labral cyst. Confirmation was noted by the presence of any of these findings in other imaging planes.

Labral tears were classified by their sites (anterosuperior, antero-inferior, postero-superior and posteroinferior) anterior and posterior lesion detected in axial, sagittal and radial planes while superior and inferior in coronal and radial planes.

3. Cartilage

Defects are identified by noting fluid signal replacing the normal intermediate signal intensity of the cartilage.

\section{Other findings}

The presence of other MRI findings such as herniation pits, acetabular cyst formation, paralabral cyst formation, and acetabular protrusion that were thought to be of relevance in femoroacetabular impingement. The radiographic interpretations were compared with the surgical findings, as hip arthroscopy is considered the gold standard for diagnosis. 


\section{Statistical analysis}

Data was analyzed using SPSS win statistical package version 21 (SPSS Inc, Chicago, IL). Numerical data were expressed as mean and standard deviation or median and range as appropriate. Qualitative data were expressed as frequency and percentage. Chi-square test (Fisher's exact test) was used to examine the relation between qualitative variables. For quantitative data, comparison between two groups was done using either student t-test or Mann-Whitney test (non-parametric t-test) as appropriate. A p-value of less than 0.05 was considered significant. The sensitivity, specificity, positive predictive value (PPV) and negative predictive value (NPV) of MR for diagnosing the acetabular labral tears were calculated, using the results of hip arthroscopy as the gold standard.

\section{RESULTS}

This study population included 29 patients with age range from 18 years to 45 years with mean age of 30.9 . Tables (1) and (2) demonstrate the age distribution among the patients, it is noticed that about $45 \%$ of the patients in our study were 31-40 years.
Table (1): Age of the patients (in years)

\begin{tabular}{|c|c|}
\hline Mean & $\mathbf{3 0 . 9}$ \\
\hline Median & 31 \\
\hline Mtandard deviation & \pm 6.7 \\
\hline Maximum & 18 \\
\hline
\end{tabular}

Table (2): Age Distribution in the study.

\begin{tabular}{|c|c|c|}
\hline Age & Frequency & Percent \\
\hline$<\mathbf{2 0}$ & 2 & $6.9 \%$ \\
\hline $\mathbf{2 1 - 3 0}$ & 11 & $37.9 \%$ \\
\hline $\mathbf{3 1 - 4 0}$ & 13 & $44.8 \%$ \\
\hline $\mathbf{4 1 - 5 0}$ & 3 & $10.3 \%$ \\
\hline
\end{tabular}

Table (3) shows sex predilection amongst the different types of femoroacetabular impingement in the study population. Out of 19 cases with cam type FAI, 14 patients were males and 5 females showing male predominance with the male gender representing about $73.7 \%$ of the cam cases.On the other hand, pincer and mixed types were found more common in females in our study, with the female gender representing $100 \%$ of the pincer cases and $71.4 \%$ of the mixed type. The relationship between sex and type of impingement was proved to be statistically significant with a $\mathrm{P}$ value $=0.005$.

Table (3): Shows the correlation between sex and type in the study population

\begin{tabular}{|c|c|c|c|c|c|c|}
\hline \multirow{2}{*}{ Type } & \multicolumn{2}{|c|}{ Cam } & \multicolumn{2}{c|}{ Pincer } & \multicolumn{2}{c|}{ Mixed } \\
\cline { 2 - 7 } & Frequency & percent & Frequency & percent & Frequency & Percent \\
\hline Male & 14 & $73.7 \%$ & 0 & $0.0 \%$ & 2 & $28.6 \%$ \\
\hline Female & 5 & $26.3 \%$ & 3 & $100 \%$ & 5 & $71.4 \%$ \\
\hline
\end{tabular}

The difference in the ages of patients with different types of FAI was of a little significant, ( $\mathrm{P}$ value $=0.033$ ). Cam type (range, 18-37 years [mean, 28.1 years \pm 5.4 years] Pincer (range, 28- 43 years [mean, 37.7 years \pm 6.8 years] Mixed (range, $30-45$ years [mean, 35.7 years \pm 4.8 years].

Table (4): Shows correlation between age and type of femoroacetabular impingement

\begin{tabular}{|c|c|c|c|c|}
\hline & $<20$ & 21-30 & $31-40$ & $>40$ \\
\hline & Frequency (\%) & Frequency (\%) & Frequency (\%) & Frequency (\%) \\
\hline Cam & $2(10.5 \%)$ & $9(47.4 \%)$ & $8(42.1 \%)$ & $0 \quad(0.0 \%)$ \\
\hline pincer & $0 \quad(0.0 \%)$ & $1 \quad(33.3 \%)$ & $0 \quad(0.0 \%)$ & $2 \quad(66.7 \%)$ \\
\hline Mixed & $0(0.0 \%)$ & $0(0.0 \%)$ & $6 \quad(85.7 \%)$ & $1 \quad(14.3 \%)$ \\
\hline
\end{tabular}

Forty-three labral tears were noted and classified by their sites. $72 \%$ of the lesions (31 lesions) are localized in the antero-superior quadrant, While $14 \%$ (6 lesions) in postero-superior, $9.3 \%$ (4 lesions) in antero-inferior and $4.7 \%$ ( 2 lesions) in postero-inferior quadrant. Table (5) compares the site of labral lesions using MRA with arthroscopy in the 29 patients.

Table (5): Shows comparison of hip arthroscopy findings with MRA findings regarding labral tears distributed by location.

\begin{tabular}{|c|c|c|}
\hline QUADRANT & MRA & ARTHROSCOPE \\
\hline Antero-superior & 30 & 31 \\
\hline Postero-superior & 5 & 6 \\
\hline Antero-inferior & 4 & 4 \\
\hline Postero-inferior & 2 & 2 \\
\hline Total & 41 & 43 \\
\hline
\end{tabular}

Six patients did not have acetabular labral tears at arthroscopy. Two of these patients had labral tears predicted by MR arthrogram. 
Table (6): Sensitivity and specificity of MRA in the assessment of labral tears distributed by locations compared with arthroscopic findings.

\begin{tabular}{|c|c|c|c|c|}
\hline QUADRANT & SENSITIVITY & SPECIFICITY & PPV & NPV \\
\hline Antero-superior & $90.9 \%$ & $77.8 \%$ & $90.9 \%$ & $77.8 \%$ \\
\hline Postero-superior & $80 \%$ & $100 \%$ & $100 \%$ & $96.3 \%$ \\
\hline Antero-inferior & $100 \%$ & $100 \%$ & $100 \%$ & $100 \%$ \\
\hline Postero-inferior & $100 \%$ & $100 \%$ & $100 \%$ & $100 \%$ \\
\hline *PPV: Positive predictive value & *NPV: Negative predictive value & & \\
\hline
\end{tabular}

Comparison of the MRA and hip arthroscopy findings noted above (regarding the identification of labral tears) showed MRA to have a sensitivity of $87.5 \%$, specificity $71.4 \%$, positive predictive value of $91.3 \%$ and negative predictive value of $62.5 \%$ (Table 7) with $\mathrm{P}$ - value of 0.0028 indicating high statistical significance.

Table (7): Sensitivity and specificity of MRA in the assessment of labral tears compared with arthroscopic findings.

\begin{tabular}{l|c|}
\hline Parameters & Labrum tear \\
\hline SENSITIVITY & $87.50 \%$ \\
\hline SPECIFICITY & $71.40 \%$ \\
\hline PPV & $91.30 \%$ \\
NPV & $62.50 \%$ \\
\hline *PPV: Positive predictive value \\
*NPV: Negative predictive value
\end{tabular}

Magnetic resonance arthrography detected that articular chondral abnormalities were observed in 12 patients $(41.3 \%)$.

\section{\% of chondral injury by MRA}

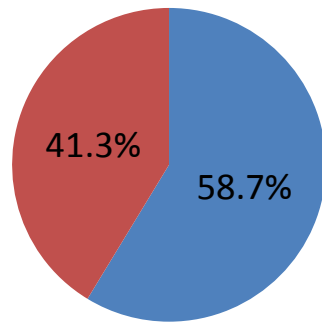

\section{Figure (1): Chart showing percentage of chondral injury by MRA.}

During arthroscopy, cartilage abnormalities were noted in 17 patients (58.6\%). Seventeen patients showed chondral abnormality at arthroscopy. Four of these patients could not be detected by MR arthrogram.

Table (8): Shows comparison of hip arthroscopy findings with magnetic resonance arthrography findings regarding the chondral abnormalities

\begin{tabular}{|c|c|c|}
\hline Chondral lesion & MRA & Arthroscopy \\
\hline Normal & 14 & $\mathbf{1 2}$ \\
\hline Degenerative & $\mathbf{1 5}$ & $\mathbf{1 7}$ \\
\hline
\end{tabular}

The results of magnetic resonance arthrography to detect chondral abnormalities,

compared with the results of hip arthroscopy, are presented in table (8). The sensitivity and specificity of detecting chondral lesions with magnetic resonance arthrography were $76.5 \%$ and 91.7\% respectively showing high statistical significance with P-value of 0.0013 .

Table (9): Shows sensitivity and specificity of MRA in the assessment of chondral Lesions compared with arthroscopic findings.

\section{\begin{tabular}{l|l}
\hline Parameters & Chondral pathology
\end{tabular}}

\begin{tabular}{|l|l|}
\hline SENSITIVITY & $76.47 \%$ \\
\hline SPECIFICITY & $91.67 \%$ \\
\hline PPV & $86.67 \%$ \\
\hline NPV & $78.57 \%$ \\
\hline
\end{tabular}

*PPV: Positive predictive value

*NPV:

Negative predictive value

Other findings noted on the MRA included labral cysts (3 patients $10.3 \%$ ) and synovial pit (3 patients $10.3 \%$ ).

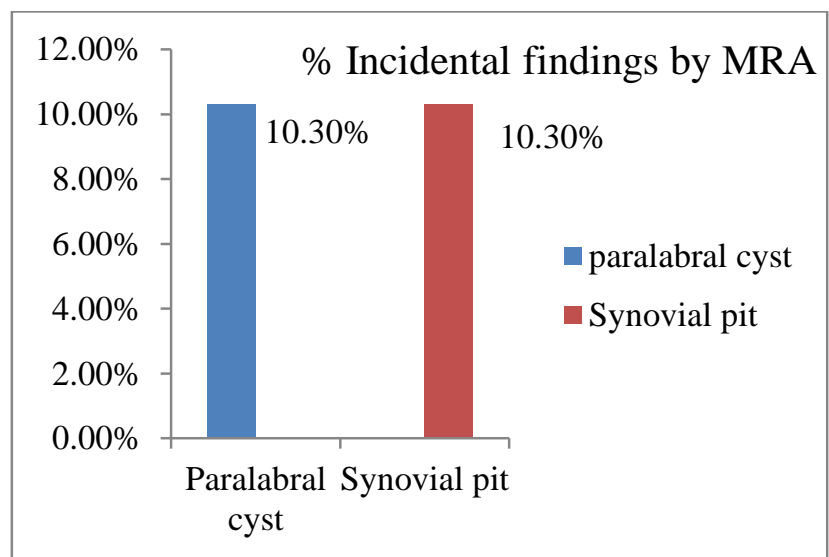

Figure (2): A diagram showing percentage of incidental findings

Collectively, our results showed relatively high sensitivity and positive predictive value of MRA (about 87.5 and $91.3 \%$ respectively) in diagnosis of hip labral lesions. However, it showed lower sensitivity $(76.47 \%)$ regarding the detection of chondral lesions. Moreover, the use of MRI enabled the detection of osseous abnormalities and early degenerative changes. 


\section{CASE PRESENTATION}

Case 1

History:

- 31 years old female complaining of right hip pain.

Findings:

Conventional MRI shows cystic changes at the anterior aspect of the femoral head-neck junction and anterior acetabular rim shows low signal in T1-weighted images and high signal in STAIR.

Evidence of abnormal neck-head junction showing increased a. angle $78.4^{\circ}$, and deep acetabulum with acetabular depth measuring(-) $2.2 \mathrm{~mm}$.
MRA shows high signal intensity linear band seen deep to anterior labrum separating it from its acetabular attachment, superior labral tear is also noted. Chondral degeneration of anterior and posterior femoral articular cartilage is noted.

Diagnosis:-

- Mixed type of FAI with anterior and superior labral tear associated with cartilage degeneration. Arthroscopy confirmed MRA diagnosis.

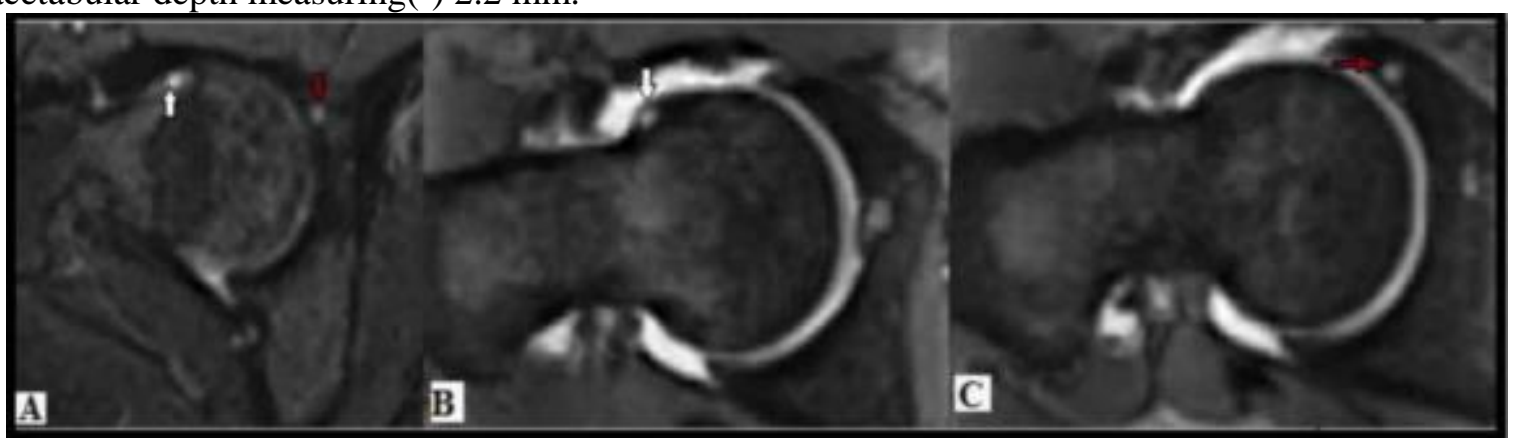

Figure (3): (A) axial STIR image, (B) and (C) T1-weighted arthrogram axial oblique images showing cystic changes at osseous bump of femur (White Arrow) and cystic changes of acetabular rim (Red Arrow).

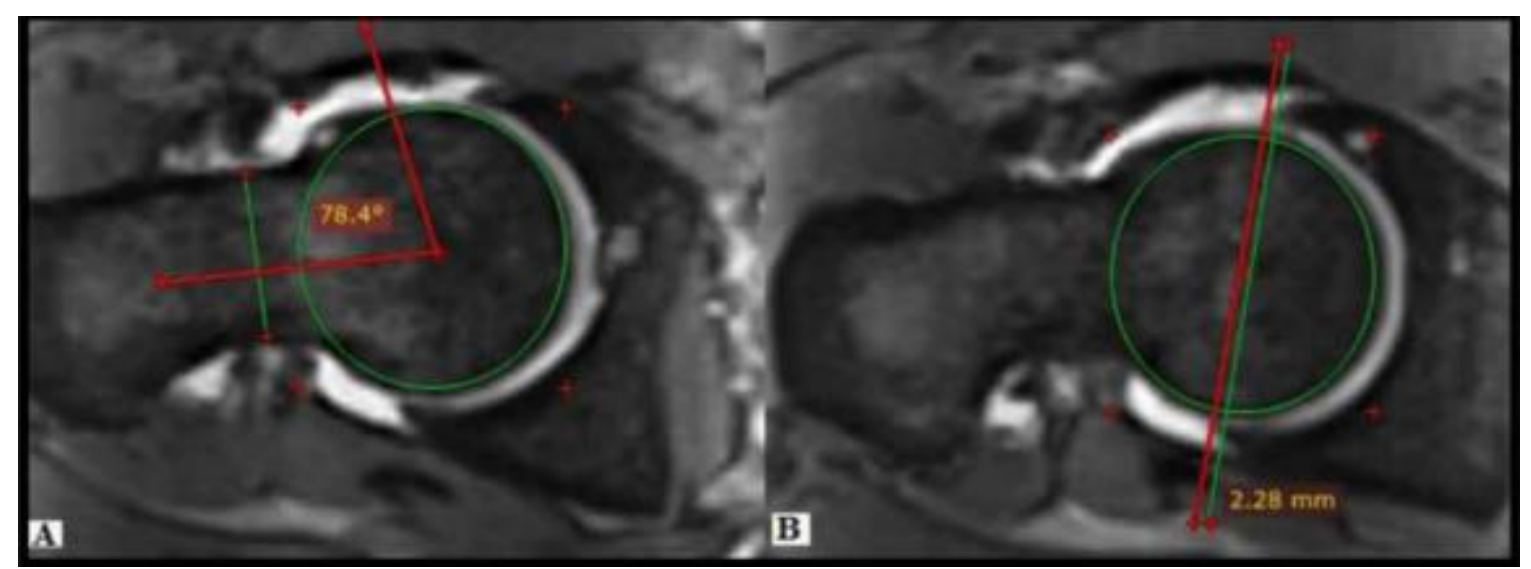

Figure (4): T1- weighted fat sat arthrogram axial oblique images showing (A) $\alpha$ angle $=78.4^{\circ}$ and (B) acetabular depth (-) $2.2 \mathrm{~mm}$.

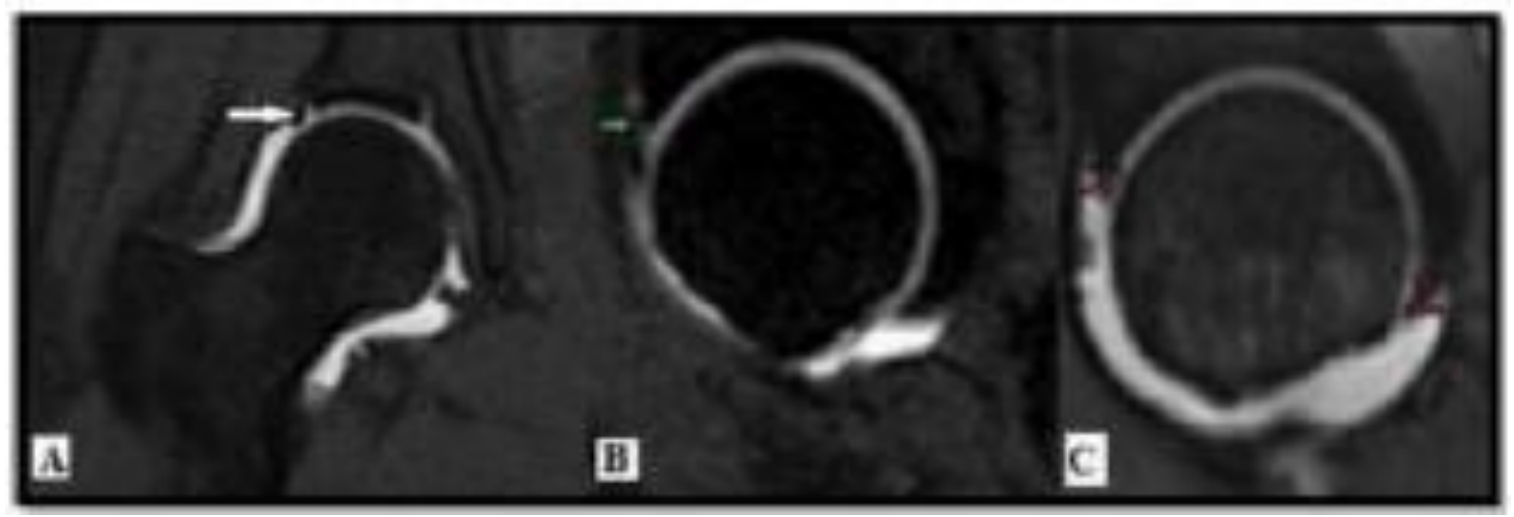

Figure (5): T1-weighted images Fat Sat (A) coronal image showing superior labral tear (white arrow). (B) Sagittal image showing anterior labral tear (Green Arrow). (C) Sagittal image showing cartilage degeneration (Small Red Arrows). 


\section{CASE 2}

\section{- History:}

24 years old male complaining left hip pain and limitation of movement for 6 months.

- Findings:

Conventional MRI shows bone marrow edema with low signal in T1-weighted images and high signal in STIR. No evidence of subchondral marrow changes or joint effusion.

\section{MRA diagnosis}

- Evidence of abnormal neck-head junction resulting in increased $\alpha$ angle $88.2^{\circ}$ and normal acetabular depth measuring $=(+) 4.61 \mathrm{~nm}$.

- MRA shows high signal intensity linear band seen deep to anterior labrum separating it from its acetabular with chondral degeneration of femoral articular cartilage at site of the tear.

- Diagnosis:

Cam type of FAI with anterior labral tear and chondral degeneration.

Arthroscopy confirmed

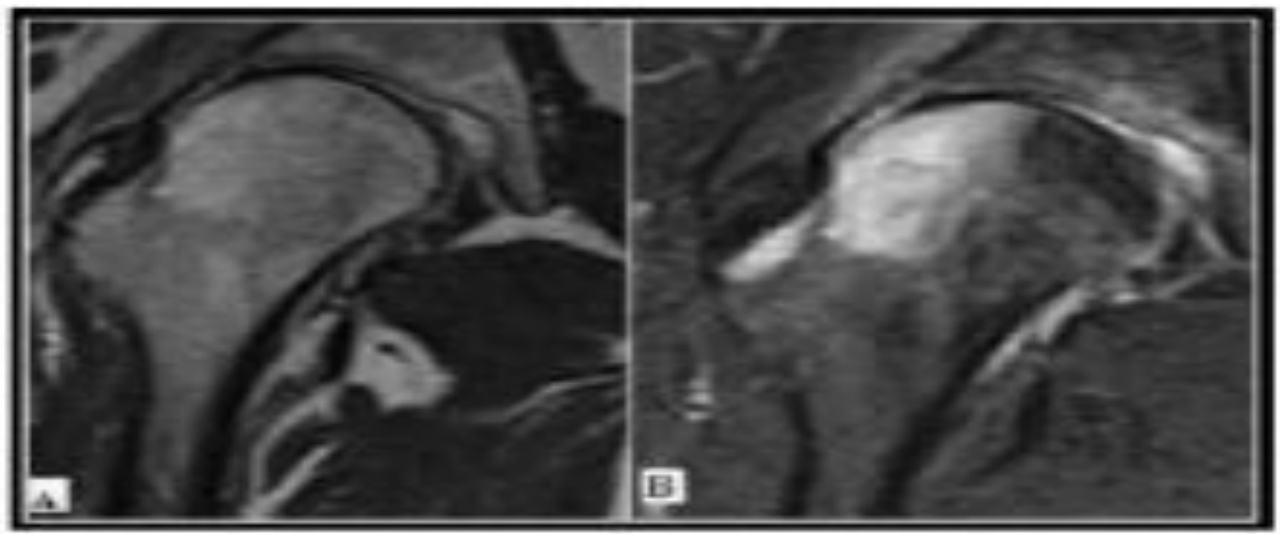

Figure (6): (A) T1-wieghted Coronal image showing low signal of bone marrow (B) T2 weighted STIR shows high signal of the bone marrow denoting marrow edema. Loss of femoral head and neck waist is noted.

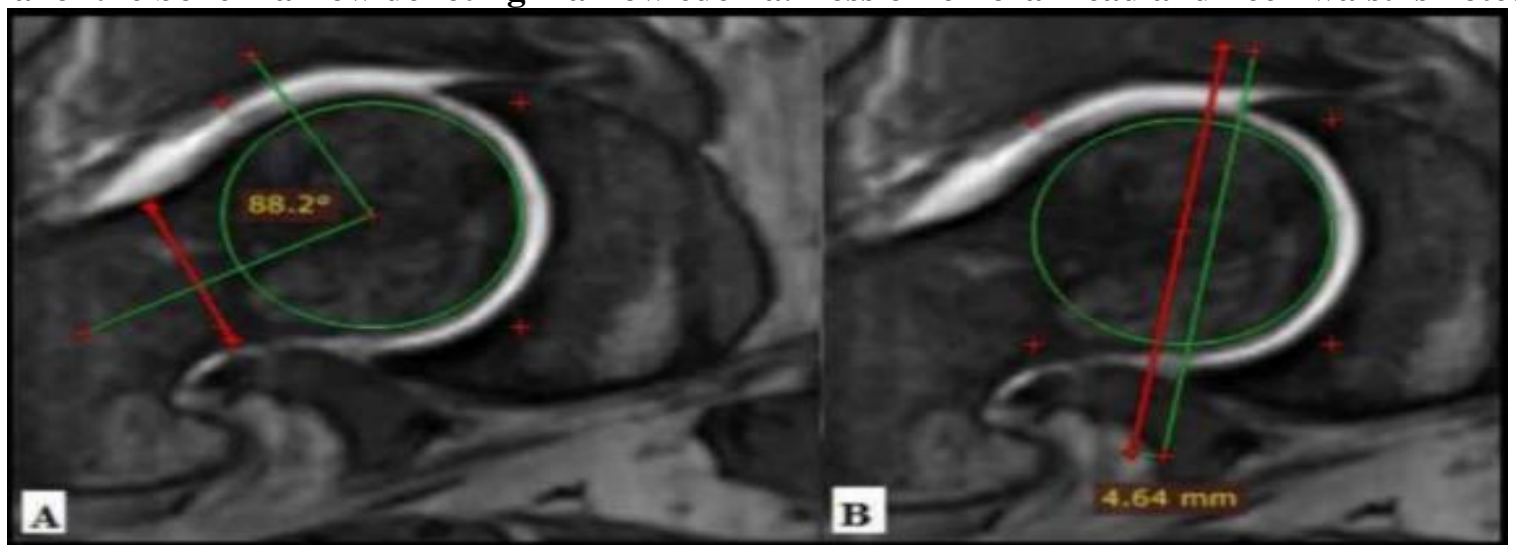

Figure (7): T1-weighted fat sat arthrogram axial oblique images showing (A) $\alpha$ angle $=88.2^{\circ}$ and (B) acetabular depth $(+) 4.6 \mathrm{~mm}$.

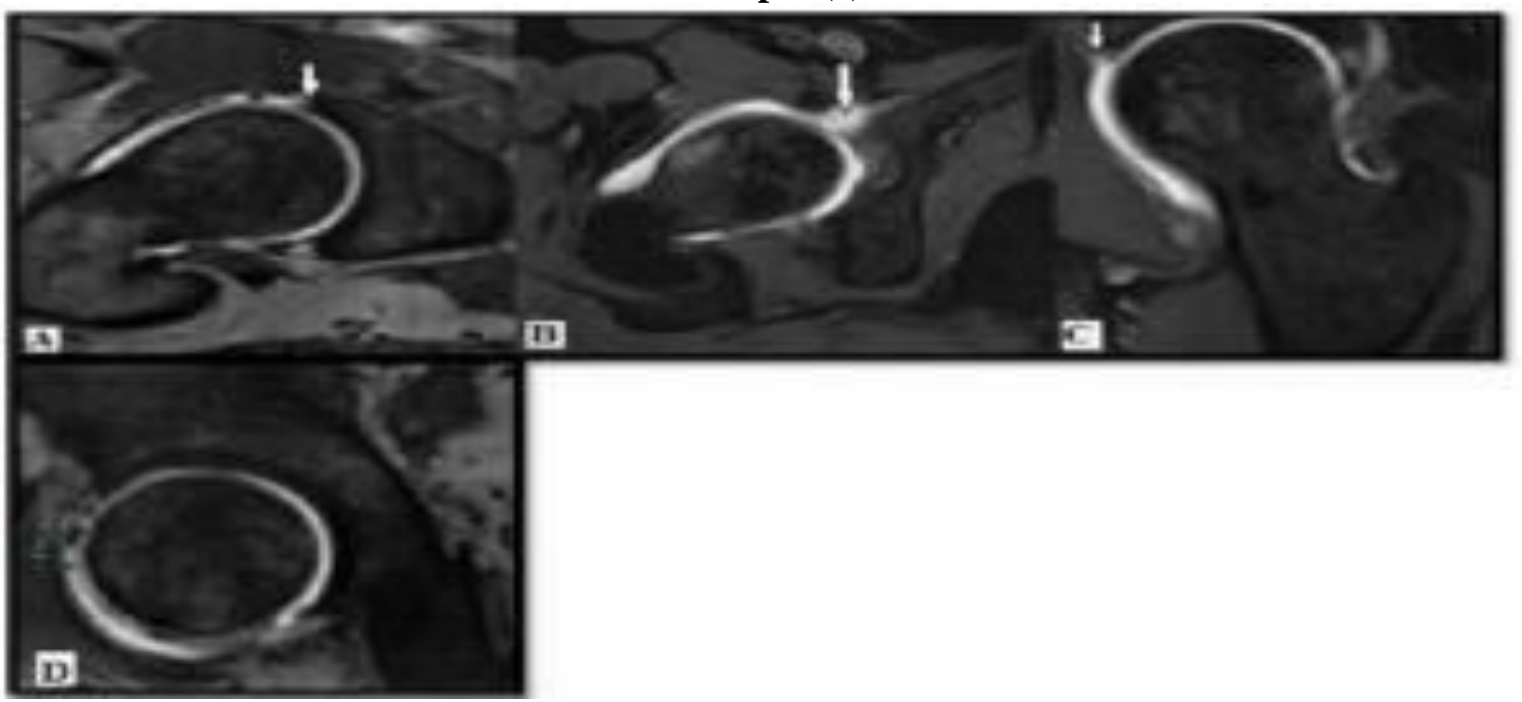

Figure (8): T1-weighted fat sat arthrogram shows (A), (B) axial and (C) radial shows anterior labral tear (White Arrow) and (D) sagittal image showing cartilage degeneration (Small Green Arrows). 


\section{- History:}

33 years old female patient complaining of left hip pain for 1 year.

- Findings:

- Conventional MRI shows no evidence of bone marrow edema, subchondral marrow changes or effusion.

- Evidence of abnormal neck-head junction and deep acetabulum is noted. The $\alpha$ angle $=$ $87.6^{\circ}$ and the acetabular depth measures (-) 1 . $7 \mathrm{~mm}$.

MRA shows high signal intensity linear band seen deep to anterior-superior labrum separating it from its acetabular attachment. Superior labral tear is also noted.

\section{- Diagnosis:}

Mixed type of FAI with anterior and superior labral tear.

- Arthroscopy confirmed MRA diagnosis.

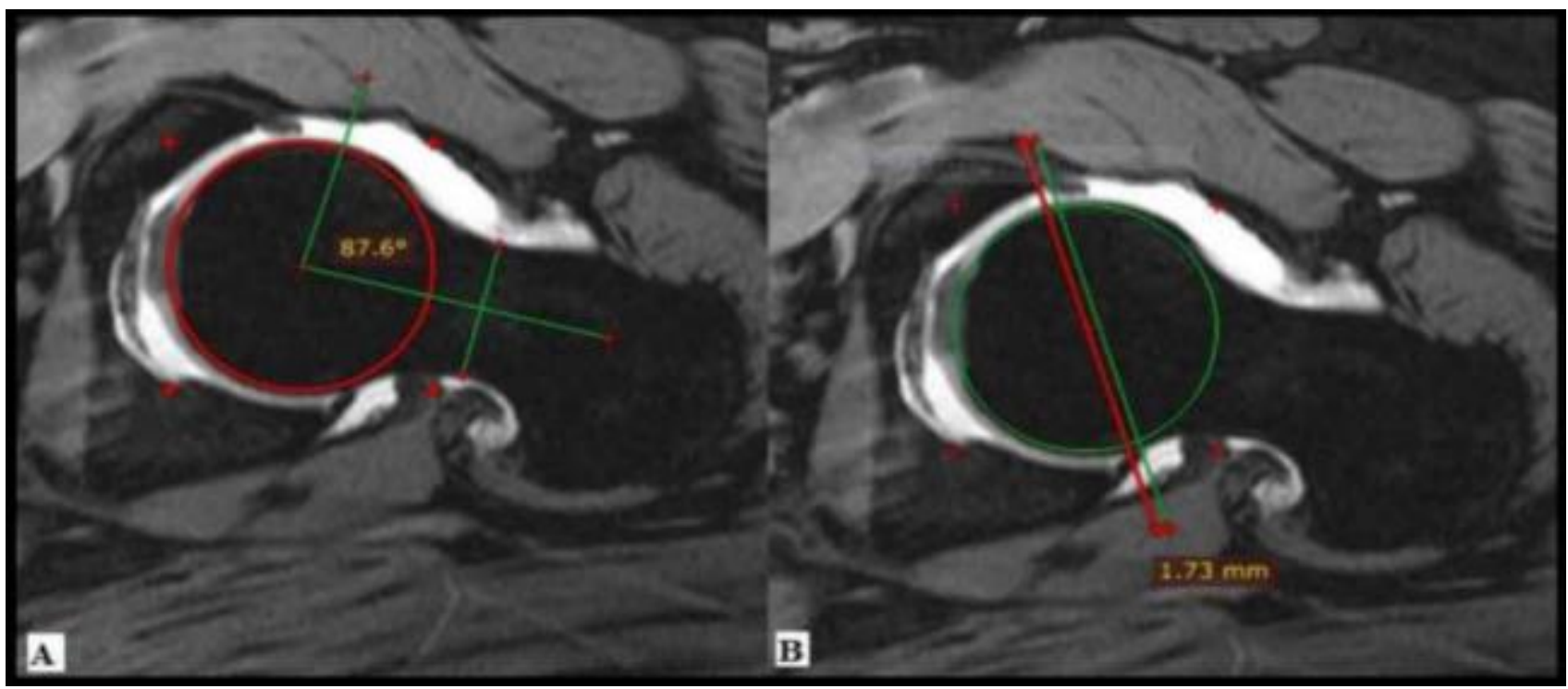

Figure (9): T1-weighted fat sat arthrogram axial oblique images showing (A) $\alpha$ angle $=87.6^{\circ}$ and (B) acetabular depth (-) $1.73 \mathrm{~mm}$.

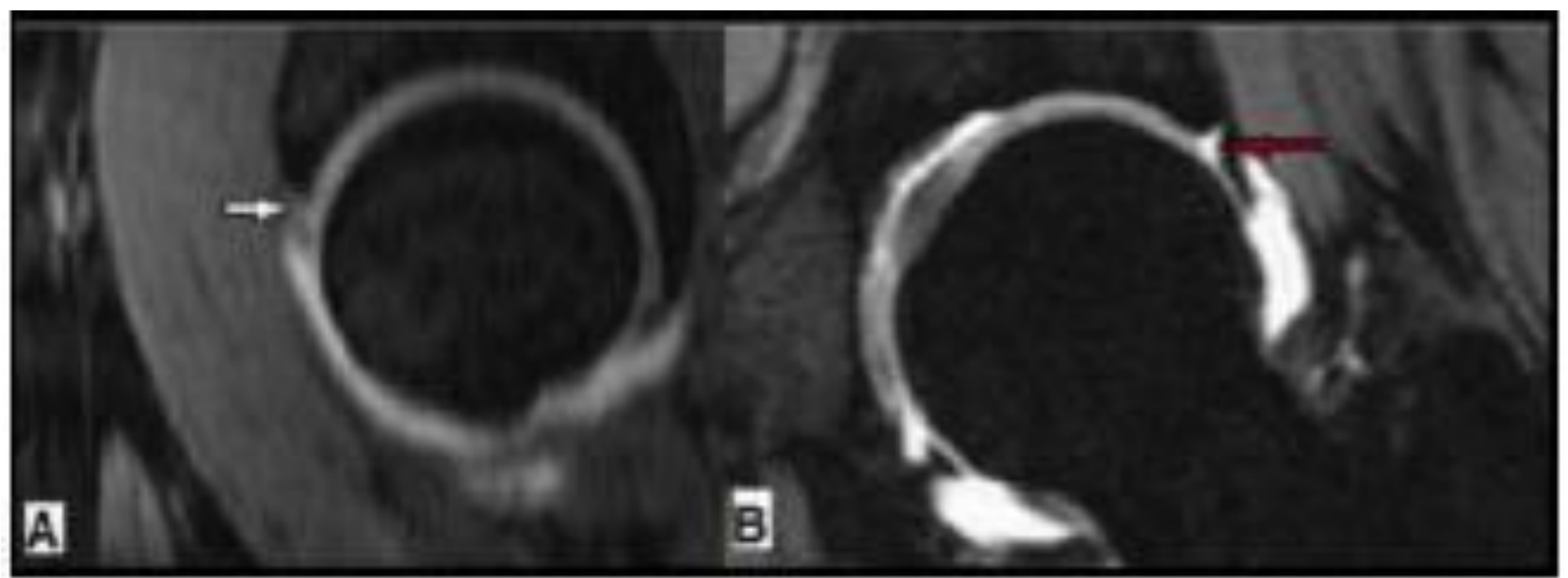

Figure (10): T1 weighted images fat sat (A) sagittal image showing anterior labral tear (white arrow) and (B) coronal images showing superior labral tear (Red Arrow). 


\section{History:}

30 years old female complaining of right hip pain and limitation of movement.

\section{- Findings:}

- Conventional MRI shows cystic changes at the posterior aspect of the femoral head with low signal intensity in T1-weighted images and high signal in T2- weighted images.

○ Evidence of abnormal neck-head junction resulting in increased $\alpha$ angle $77.4^{\circ}$, and deep
○ acetabulum with acetabular depth measuring (-) $3.1 \mathrm{~mm}$.

MRA shows high signal intensity linear band seen deep to anterior and superior labrum separating it from its acetabular attachment. Chondral degeneration of posterior femoral articular cartilage is noted.

- Diagnosis:

Mixed type of FAI with anterior and superior labral tear associated with cartilage degeneration.

- Arthroscopy confirmed MRA diagnosis.

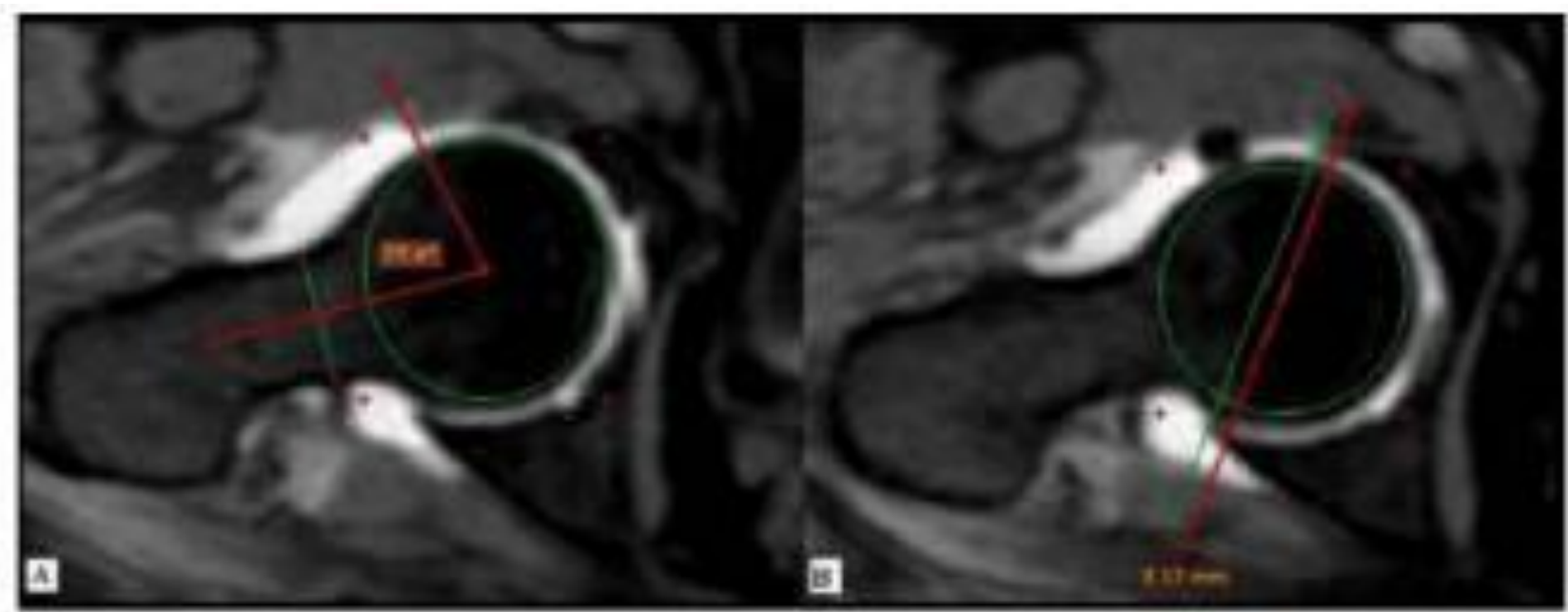

Figure (11): T1-weighted fat sat arthrogram axial oblique images showing (A) $\alpha$ angle $=77.4^{\circ}$ and (B) acetabular depth (-) $3.1 \mathrm{~mm}$.
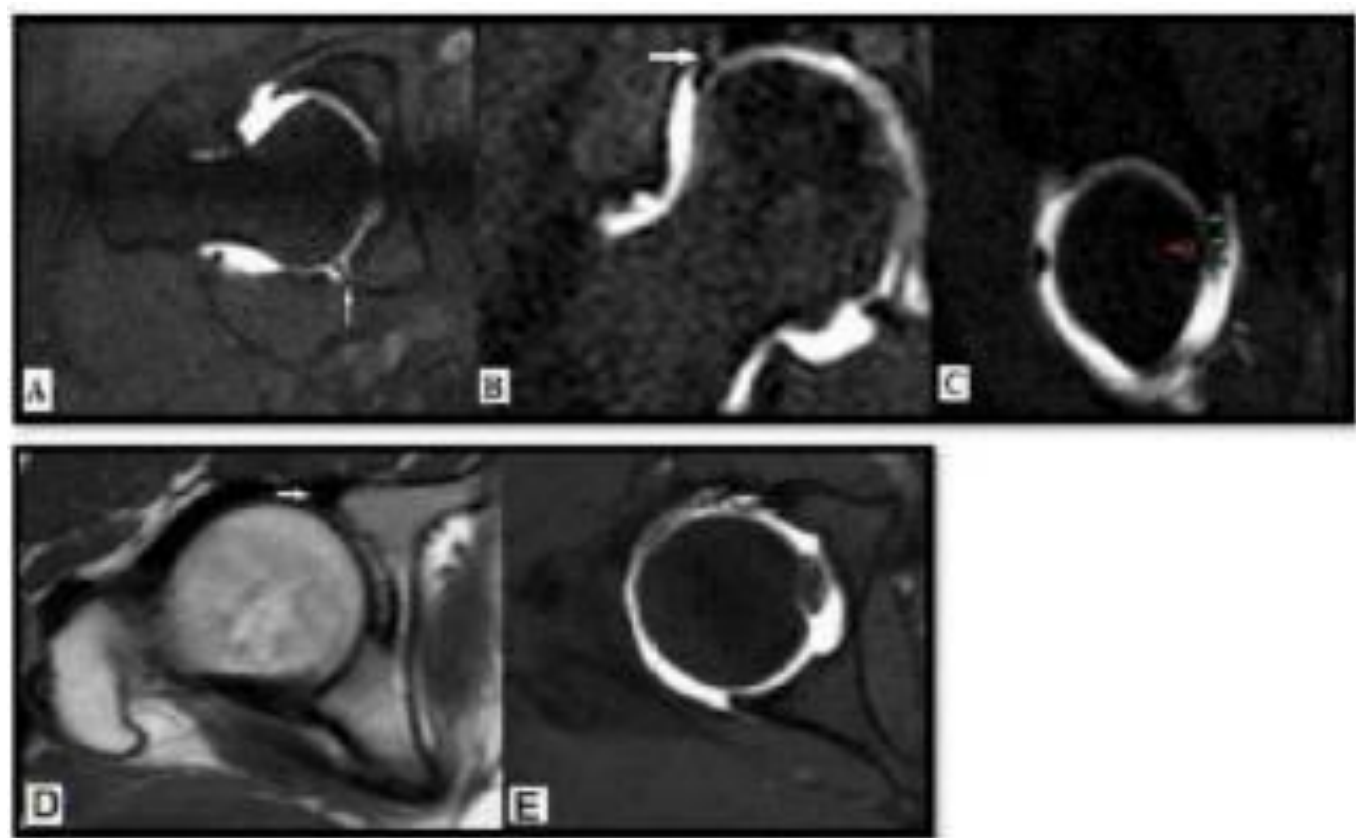

Figure (12): T1-weighted images fat sat (A) radial image showing anterior labral tear (White Arrow) (B) coronal image showing superior labral tear (White Arrow) (C) sagittal image showing cartilage degeneration (Small Green Arrows) and cystic changes is seen at posterior aspect of femoral head (Red Arrow). (D) Conventional MRI T1weighted axial plane showing no evidence of labral tear. (E) T1- weighted FS arthrogram showing anterior labral tear could not be detected by conventional MRI. 
ejhm.journals.ekb.eg

\section{DISCUSSION}

The purpose of this study was to assess the diagnostic role of MRA in the detection of labral and cartilaginous abnormalities in patients with femoroacetabular impingement syndrome and comparing its diagnostic value with arthroscopy.

The study included 29 patients, 16 males and 13 females, their age ranged from 18 to 45 years and the mean age was 30.9 years with standard deviation of \pm 6.7. This matched the study done by Farkas et al. ${ }^{(10)}$, which included 20 patients of FAI, 6 males and 14 females. Their age range was 17 to 46 years and the mean age was 31.5 years old with standard deviation of \pm 8.4 .

Three types of morphologic abnormalities can occur in FAI: cam, pincer, and mixed. Cam deformity is characterized by an abnormal/aspherical morphology of the proximal femur. Pincer deformity is characterized by focal or general overcoverage of the femoral head by the acetabulum. The third type of FAI, mixed, is a combination of cam and pincer impingement characteristics ${ }^{(\mathbf{1 1})}$.

In this study, the cam type was the most common. Nineteen out of 29 patients $(65.5 \%)$ were cam, seven patients $(24.1 \%)$ were mixed and three patients $(10.3$ $\%)$ were pincer type.

In consistence with our study, Tresch et al. ${ }^{(12)}$ found in their study, that cam was more common among their study sample, as 33 out of 63 patients were cam, 20 patients were mixed and 10 patients were pincer type.

However, in contrast to our study, Genovese et al. ${ }^{(7)}$, Banerjee et al. ${ }^{(8)}$ and Sangal et al. ${ }^{(13)}$, stated that combination of cam and pincer lesions (mixed type) is the most common presentation of FAI in general population and isolated forms are very uncommon.

Regarding sex predilection, our study showed cam type to be more common in males (out of the 19 cam patients, 14 were males and 5 were females), while the pincer and mixed types were more common in females (all three patients showing pincer type were females and out of the seven mixed patients, five were females and only 2 were males).

Similar sex distribution was demonstrated by the studies made by Amanatullah et al. ${ }^{(2)}$, Banerjee et al. ${ }^{(8)}$ and Pfirrmann et al. ${ }^{(14)}$ who declared that cam type was more common in males and pincer type was more common in females.

In our study, the cam type was common in age range of 21-30 years ( 9 out of 22) (47.4\%) while pincer type was more common in age range of $>40$ years. Mixed type was more common in age range of 31-40 years old. Similarly, Anderson et al. ${ }^{(15)}$ found in their study that cam type was more common in age range of 20-30 years. But in contrary to our study, Anderson et al. mentioned that, pincer type was more common in age range of 30-40 years. However, no specific age group for the mixed type was specified in literature.
MRA may have an expanding role in the evaluation of mild osseous abnormalities of the hip, especially in cases with mild hip deformity such as variations of acetabular retroversion and decreased femoral headneck offset, where standard radiographic images taken slightly out of rotation from the plane of reference may be suboptimal. These changes may be more clearly defined on MR images because of the ability to assess for deformity in multiple planes of reference ${ }^{(\mathbf{1 6})}$.

Using MRA, $89.6 \%$ of the cases examined in our study showed detectable osseous abnormalities; 18 out of 29 patients (62\%) showed (osseous bump), 8 patients showed loss of femoral neck waist (27.6\%) compared to the study done by James $\boldsymbol{e t}$ al. ${ }^{(\mathbf{1 7 )}}$, which included 46 patients, who found that 18 patients (39.1\%) showed osseous anatomical bony changes (osseous bump) and two patients $(4.3 \%)$ with femoral waist deficiency.

Our study used alpha-angle measurement to classify the examined cases into cam, pincer and mixed type impingement. Alpha angle was above $55^{\circ}$ in cam and mixed patients with mean $73^{\circ} \pm 7.8$, however in pincer type the angle was $<55^{\circ}$ with mean $52^{\circ} \pm 1.1$.

Acetabular depth is the distance between a line connecting both acetabular rims and the line passing through the center of the femoral head equals the acetabular depth, with the value being positive $(+)$ if the center of the femoral head projects lateral to the line connecting the acetabular rims. Negative values (-) indicate deep acetabulum ${ }^{(\mathbf{1 8})}$.

In our study the acetabulum was abnormally deep (negative value), in pincer type (three patients) and mixed type (seven patients). However, the acetabular depth showed (positive value) in cam type (19 patients), this matches Pfirrmann et al. ${ }^{(14)}$ study who found that the acetabulum was deeper in the pincer FAI group than cam FAI group.

Reports in the radiology literature suggested that the use of magnetic resonance arthrography improved the capability to detect a spectrum of intra-articular hip pathologies. MRA has excellent sensitivity and specificity with regard to the evaluation of intraarticular pathology specifically allowing unproved detection of acetabular labral tears (16).

A meta-analysis by Smith et al. ${ }^{(19)}$ suggested that MRA appears to be superior to conventional MRI for detecting an acetabular labral tear, considering the inability of conventional MRI to detect small tears and the difficulty in determining if intra-labral signal abnormalities extended to the surface of the labrum. They also suggested that conventional MRI was unable to distinguish between degeneration of the labrum, labral tear or complete detachment of the labrum from the acetabular rim compared with MRA. This is of vital importance as such variations would have a significant effect on an orthopedic surgeon's clinical decisionmaking considering surgical intervention ${ }^{(\mathbf{1 9})}$. Accordingly, assessment of acetabular labral pathology in our study series was done using MRA. 
Regarding the presence of labral tears, our study detected labral tears in 22 cases, while seven cases showed no evidence of labral tears on MRA. On the other hand, arthroscopy (the gold standard for labral assessment) detected labral tears in 23 cases, while six cases showed no evidence of labral tears, resulting in sensitivity of $87.5 \%$, specificity of $71.4 \%$, PPV of 91.3 $\%$, and NPV of $62.5 \%$.

This agreed with a meta-analysis by James et al. ${ }^{(17)}$ who evaluated the diagnostic value of MRA versus arthroscopic reference standard in 19 studies. The sensitivity for detection of labral tears ranged from $69 \%$ to $100 \%$, with most studies showing sensitivity greater than $90 \%$, and the specificity for detection of labral tears was far more variable ranging from zero to $100 \%$, with most studies showing specificity less than $80 \%$. Similarly, in Smith et al. (19) analysis of 19 studies assessing the sensitivity and specificity of MRA, MRA demonstrated a sensitivity of $87 \%$ and specificity of 64\%. Pfirrmann et al. (14) who used 3 Tesla MRA showed sensitivity slightly higher than our study reaching about $90 \%$, specificity $84.62 \%$, PPV $90 \%$ and NPV $84.62 \%$.

Changes secondary to FAI are not just limited to the acetabular labrum and articular cartilage but can also be seen in the proximal femur and bony acetabulum. Such abnormalities include the development of subchondral edema and juxta-articular fibrocystic changes usually at the site of the dysplastic femoral bump ${ }^{(20)}$.

In our study, $31 \%$ (9 patients) showed presence of subchondral marrow changes (marrow edema and/or subchondral cystic changes), compared to a study done by James et al., 2006 ${ }^{(17)}$ that included 46 patients with $37 \%$ (17 patients) with subchondral marrow changes

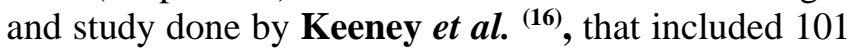
patients with $23 \%$ (24 patients) showed subchondral marrow changes.

In our experience, MRA can be a valuable tool in assisting the evaluation of osseous sources of femoroacetabular impingement as well as secondary labral pathology and degenerative changes. However, it showed limited sensitivity in detecting cartilage defects.

Collectively, MRA provide valuable information in selecting appropriate surgical management approaches and optimizing treatment of patients with intra-articular sources of hip pain.

Our study strengthens the already popular use of MRA as the imaging method of choice in detecting labral and chondral lesions in femoroacetabular impingement syndrome

\section{CONCLUSION}

- In our experience, conventional MRI and MRA are accurate methods for evaluation of both structural abnormalities and pathologic changes associated with femoroacetabular impingement.

- MRA appears to be an efficacious imaging modality in the evaluation of labral tears with a relatively high sensitivity and PPV.
- MRA is less efficacious in the diagnosis of cartilage abnormalities in the hip, both femoral and acetabular cartilage due to limited diagnostic sensitivity. Suggesting that at present MRA possesses only moderate accuracy for detecting cartilage lesions.

- The detection of acetabular labral tears and other intraarticular pathology assists the surgeon and patient in confirming the decision to proceed with arthroscopic management of articular hip pain.

- MRI is an excellent method of preoperative planning and helps guide the surgeon in localizing labral and chondral abnormalities. Furthermore, MRI may enable the referring surgeon to decide preoperatively which patients may require an arthroscopic versus an open surgical technique.

\section{REFERENCES}

1. Amanatullah, DF, Antkowiak T, Pillay $K$ et al. (2013): Prevalence of cam and pincer-type deformities on hip MRI in an asymptomatic young Swiss female population: a crosssectional study. Osteoarthritis and Cartilage, 21(4): 544-550.

2. Amanatullah DF, Antkowiak T, Pillay $\mathrm{K}$ et al. (2013): Femoroacetabular impingement: current concepts in diagnosis and treatment. Orthopedics, 38(3):185-199.

3. Tanzer M and Noiseux N (2004): Osseous abnormalities and early osteoarthritis: the role of hip impingement. Clinical Orthopaedics and Related Research, 429: 170-177.

4. Ito K, Leunig M and Ganz R (2004): Histopathologic features of the acetabular labrum in femoroacetabular impingement. Clinical Orthopaedics and Related Research, 429: 262-271.

5. Reichenbach S, Leunig $M$, Werlen $S$ et al. (2011): Association between cam type deformities and magnetic resonance imaging-detected structural hip damage: A cross sectional study in young men. Arthritis \& Rheumatism, 63(12): 4023-4030.

6. Meyer DC, Beck M, Ellis T et al. (2006): Comparison of six radiographic projections to assess femoral head/neck asphericity. Clinical Orthopaedics and Related

Research, 445: 181-185.

7. Genovese E, Spiga S, Vinci $\mathrm{V}$ et al. (2013): Femoroacetabular impingement: role of imaging. Musculoskeletal Surgery, 97(2):117-126.

8. Banerjee $P$ and Mclean CR (2011): Femoroacetabular impingement: a review of diagnosis and management. Current Reviews in Musculoskeletal Medicine, 4(1): 23-32.

9. Anderson LA, Peters CL, Park BB et al. (2009): Acetabular cartilage delamination in femoroacetabular impingement. J Bone Joint Surg Am., 91(2): 305-313.

10. Farkas GJ, Cvetanovich GL, Rajan KB et al. (2015): Impact of Femoroacetabular Impingement Morphology on Gait Assessment in Symptomatic Patients. Sports Health: A Multidisciplinary Approach, 7(5): 429-436.

11. Pun S, Kumar D and Lane NE (2015): Review: femoroacetabular impingement. Arthritis \& Rheumatology, 67(1): 17-27.

12. Tresch F, Dietrich TJ, Pfirrmann CW et al. (2016): Hip MRI: Prevalence of articular cartilage defects and labral tears in asymptomatic volunteers. A comparison with a matched population of patients with femoroacetabular impingement. Journal of Magnetic Resonance Imaging, 46(2):440-451. 
13. Sangal RB, Waryasz GR and Schiller JH (2014): Femoroacetabular impingement: a review of current concepts. Rhode Island Medical Journal, 97(11): 97:33-38.

14. Pfirrmann CW, Mengiardi B, Dora C et al. (2006): Cam and Pincer Femoroacetabular Impingement: Characteristic MR Arthrographic Findings in 50 Patients 1. Radiology, 240(3): 778-785.

15. Anderson CN, Riley GM, Gold GE et al. (2013): Hipfemoral acetabular impingement. Clinics in Sports Medicine, 32(3): 409-425.

16. Keeney JA, Peelle MW, Jackson J et al. (2004): Magnetic resonance arthrography versus arthroscopy in the evaluation of articular hip pathology. Clinical Orthopaedics and Related Research, 429: 163-169.
17. James SL, Ali K, Malara F et al. (2006): MRI findings of femoroacetabular impingement. American Journal of Roentgenology, 187(6): 1412-1419.

18. Keck J, Kienle KP, Siebenrock KA et al. (2012): September. Overcoverage of the Hip in Femoroacetabular Impingement: MRI-based Study in Patients with Radiographic Classified Pincer Type Impingement. In Orthopaedic Proceedings, 94: 426-426.

19. Smith TO, Hilton G, Toms AP et al. (2011): The diagnostic accuracy of acetabular labral tears using magnetic resonance imaging and magnetic resonance arthrography: a metaanalysis. European Radiology, 21(4): 863-874.

20. Filigenzi JM and Bredella MA (2008): MR imaging of femoroacetabular impingement. Applied Radiology, 37(4): 12-16. 